\title{
Comparison of recoil effects in graphite as observed by photoemission, electron scattering, and neutron scattering
}

\author{
M. Vos, ${ }^{1}$ M. R. Went, ${ }^{1}$ Y. Kayanuma, ${ }^{2}$ S. Tanaka,${ }^{3}$ Y. Takata, ${ }^{4}$ and J. Mayers ${ }^{5}$ \\ ${ }^{1}$ Atomic and Molecular Physics Laboratories, Research School of Physical Sciences and Engineering, The Australian National \\ University, Canberra ACT 0200, Australia \\ ${ }^{2}$ Graduate School of Engineering, Osaka Prefecture University, Sakai, Osaka 599-8531, Japan \\ ${ }^{3}$ Graduate School of Sciences, Osaka Prefecture University, Sakai, Osaka 599-8531, Japan \\ ${ }^{4}$ RIKEN SPring-8 Center, Sayo-cho, Hyogo 679-5148, Japan \\ ${ }^{5}$ ISIS Facility, Rutherford Appleton Laboratory Chilton, Didcot, OX11 OQX, United Kingdom
}

(Received 21 April 2008; revised manuscript received 25 May 2008; published 2 July 2008)

\begin{abstract}
Recoil effects have been observed for neutron and electron scatterings, and in photoemission. In all cases highly oriented pyrolytic graphite was used as a testing material and dependencies of recoil effects on the crystal orientation were found but these results have, so far, not been compared. We show that the same theory can describe the results of all three experiments in a quantitative way.
\end{abstract}

DOI: 10.1103/PhysRevB.78.024301

PACS number(s): 79.60.-i, 78.70.Nx, 79.20.Kz

\section{INTRODUCTION}

There is a range of experiments where a well-defined amount of momentum is transferred to a single atom in a lattice. We will consider here neutrons with several electron volts of energy scattered over large angles, $\mathrm{keV}$ electrons deflected by an atom, or core electrons emitted after absorption of a high-energy photon. Due to this momentum transfer, the atom will start moving and thus acquire kinetic energy. For a free, stationary atom (mass $M$ ), the recoil energy $E_{r}^{f, 0}$ is given by $E_{r}^{f, 0}=q^{2} / 2 M$ with $\boldsymbol{q}$ as the transferred momentum. If a free atom has a momentum $\boldsymbol{k}$ before the collision then the recoil energy is the difference between its kinetic energy before and after the collision,

$$
E_{r}^{f, \boldsymbol{k}}=\frac{(\boldsymbol{k}+\boldsymbol{q})^{2}}{2 M}-\frac{k^{2}}{2 M}=\frac{q^{2}}{2 M}+\frac{\boldsymbol{q} \cdot \boldsymbol{k}}{M} .
$$

The energy of the detected particle is reduced by this amount. Thus for scattering from an ensemble of free, moving particles, the recoil energy distribution shows a Compton profile of their momentum distribution.

Very recently a paper was published showing recoil effects in high-energy photoemission experiments on graphite. ${ }^{1}$ Neglecting the momentum of the photon, the carbon recoil $\boldsymbol{q}$ is simply $\boldsymbol{- P}$ with $\boldsymbol{P}$ as the momentum of the photoelectron. The recoil causes an apparent shift to higher binding energy of the core level, as well as additional broadening. Similarly recoil effects have been observed for elastic scattering of $\mathrm{keV}$ electrons over large angles from graphite and the recoil effect causes a reduction in their energy as well as broadening. ${ }^{2}$ Recoil effects also play a role in neutron scattering and results for graphite were published by several authors. ${ }^{3-5}$ In these cases the scattering is not from a free atom but from an atom bound to a lattice. Hence the simple picture, sketched above, needs modifications. In this paper we will show that we can interpret all these measurements in terms of the vibrational properties of the lattice in a uniform way.

How does the recoil energy change if the atom is bound to a lattice? Lamb was the first to discuss this for the case of resonant absorption of a neutron. ${ }^{6}$ Here an incoming neutron with kinetic energy $E$ and mass $m$ is absorbed by an atom. This experiment is in many ways the inverse of the highenergy photoemission experiment where the cause of the recoil is the emission of a particle rather than its absorption. $E_{0}$ is the energy that the neutron would have at resonance if the compound nucleus did not take up kinetic energy. For a stationary and free atom, the cross section is then given by

$$
\sigma(E)=\frac{\Gamma^{2}}{4} \frac{\sigma_{0}}{\left(E-E_{0}-E_{r}^{f, 0}\right)^{2}+\frac{1}{4} \Gamma^{2}},
$$

with $\Gamma$ as the width of the resonance. For a free atom with mass $M \gg m$, the recoil energy is $\approx \frac{m}{M} E$. In practice the absorbing atom is bound to a lattice. Thus by measuring $\sigma(E)$, one obtains information about the distribution of $E_{r}^{c}$ : the recoil energy distribution of an atom bound in a crystal lattice. Lamb concluded that, if $q$ is large enough, one observes a recoil energy distribution that resembles that of neutrons absorbed by free particles with a momentum distribution $n(\boldsymbol{p})$. At high temperatures $n(\boldsymbol{p})$ is that of a classical gas with the same temperature but at low temperatures $n(\boldsymbol{p})$ resembles that of a classical gas with a temperature of the order of the Debye temperature of the sample. He also sketched the expected spectra at low and intermediate $\boldsymbol{q}$ values.

Later, scattering experiments using neutrons with epithermal energies became possible and the recoil energy distribution was studied in more detail. ${ }^{7-11}$ The picture emerging from these $\left(n, n^{\prime}\right)$ experiments confirmed the conclusion of Lamb. Again, if $q$ is large enough, one can interpret the measurement in terms of the momentum distribution of the target atoms. ${ }^{12}$ Hence the term neutron Compton scattering was coined. This momentum distribution resembles that of a classical gas at high temperatures but is related to the phonon density of states at low temperatures. ${ }^{13}$ Theories were developed describing experiments for which the momentum transfer is not large enough to interpret $E_{r}$ as a Compton profile. Then the experiment can be described by adding final-state corrections to the Compton profiles. ${ }^{9}$ The term "final state 

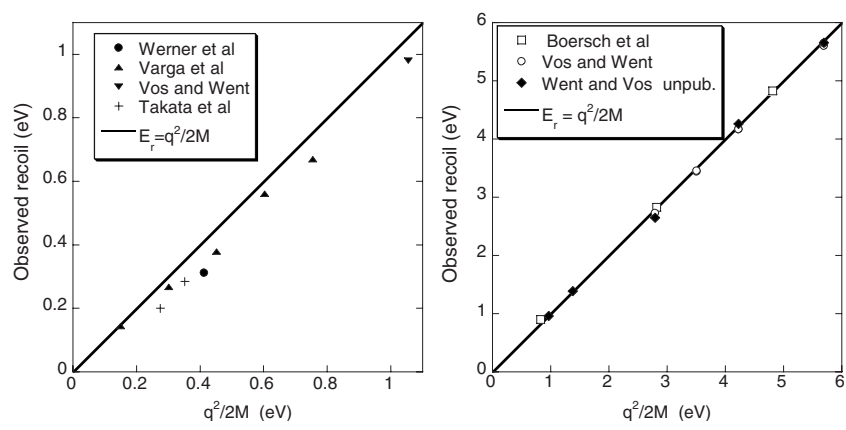

FIG. 1. The observed recoil energy compared to the expected recoil energy for scattering from a free atom for electrons scattered from carbon $[\square$ (Ref. 14), (Ref. 19), $\boldsymbol{\Delta}$ (Ref. 15), $\boldsymbol{\nabla}$ (Ref. 2), and $\bigcirc$ Ref. 20)] as well as that observed in photoemission (+) (Ref. $1)$.

corrections" refers to the fact that after the collisions the atoms cannot be considered as free particles.

The first observation of such phenomena outside neutron physics is found in the work of Boersch et al. ${ }^{14}$ They analyzed the energy of $30 \mathrm{keV}$ electrons scattered from carbon over $45^{\circ}, 90^{\circ}$, and $135^{\circ}$, and found that the energy loss in these $\left(e, e^{\prime}\right)$ experiments implied that they scattered from a single carbon atom. However they observed also a remarkable large peak width, which was attributed to heating of the sample due to the relatively intense electron beam. However, more recent work using much smaller beams also observe a large peak width related more closely to the carbon Debye temperature than the sample temperature. ${ }^{2,15}$ The recoil effect has been used to study compounds $^{16}$ and substrate-overlayer ${ }^{17}$ systems by electron scattering.

The fact that the recoil effect should influence the observed peak position in photoemission was recognized right at the beginning ${ }^{18}$ but the effect was then too small to be resolved. Recently, with the development of high-energy and high-resolution photoemission [or $(\gamma, e)]$ experiments, it has become possible to clearly resolve these effects, as was demonstrated by Takata et al. ${ }^{1}$ In this work the carbon $1 s$ photoemission spectrum was measured employing $\mathrm{x}$ rays up to 8 $\mathrm{keV}$. Recoil effects are evident from a shift to higher binding energy of the $\mathrm{C} 1 s$ peak with increasing photon energy, as well as a gradual (asymmetric) broadening of the core line with increasing photon energy. A summary of the recoil shifts for carbon, as seen by various techniques, is given in Fig. 1. All techniques have been applied to highly oriented pyrolytic graphite (HOPG) ${ }^{1-5}$ It is very anisotropic and resolving this anisotropy is a benchmark test. This paper investigates if the results of the $\left(n, n^{\prime}\right),\left(e, e^{\prime}\right)$, and $(\gamma, e)$ experiments are consistent.

\section{THEORY}

In the interpretation of these experiments, one relies on the first Born approximation. ${ }^{7}$ This is an excellent approximation for neutron scattering and should be reasonable for energetic electrons. The observed intensity is then proportional to the product of two factors,

$$
\frac{d^{2} \sigma}{d \Omega d \varepsilon}=A S(\boldsymbol{q}, \omega)
$$

The factor $A$ depends on the experimental geometry and the projectile-target interactions, and determines the intensity, not the shape, of the observed spectrum. The second factor is the dynamical structure factor $S(\boldsymbol{q}, \omega)$, which depends only on properties of the target. Thus if this approach is valid, the shape of the observed distribution, as a function of recoil energy (written here by convention as $\omega$ ) taken at the same momentum transfer, should be the same for all techniques (besides trivial factors such as experimental resolution and, for photoemission, lifetime broadening of the core level).

The dynamical structure factor is given by

$$
S(\mathbf{q}, \omega)=\frac{1}{2 \pi} \int_{-\infty}^{\infty} e^{-i \omega t} F(\boldsymbol{q}, t) d t,
$$

with

$$
F(\boldsymbol{d}, t)=\frac{1}{N} \sum_{i, j=1}^{N}\left\langle e^{-i \boldsymbol{q} \cdot \boldsymbol{r}_{i}(0)} e^{\boldsymbol{q} \cdot \boldsymbol{r}_{j}(t)}\right\rangle,
$$

with $r_{i}(t)$ as the position of atom $i$ at time $t$. If $q \gg \frac{2 \pi}{d}$ with $d$ as the nearest-neighbor distance then the terms with $i \neq j$ (corresponding to the interference of contributions from different atoms) are rapidly oscillating and averages out to zero (incoherent approximation). Thus we have to evaluate only the terms with $i=j$,

$$
F(\boldsymbol{q}, t)=\left\langle e^{-i \boldsymbol{q} \cdot \boldsymbol{r}(0)} e^{\boldsymbol{q} \cdot \boldsymbol{r}(t)}\right\rangle .
$$

In the limit of high-momentum transfer $(q \Rightarrow \infty)$, as explained by Sears, ${ }^{9}$ Eq. (6) depends only on $\mathbf{v}(0)$, the velocity distribution of the particles in the target system just before the collision. In that limit he showed that one can write for $S(\mathbf{q}, \omega)$ (in atomic units)

$$
S_{\infty}(\boldsymbol{q}, \omega)=\frac{M}{q} J(y),
$$

with $y=\frac{m \omega}{q}-\frac{q}{2}$, and $J(y)$ as the projection of the momentum density of the target atoms on the momentum-transfer direction,

$$
J\left(p_{z}\right)=\int_{-\infty}^{\infty} \int_{-\infty}^{\infty} n\left(p_{x}, p_{y}, p_{z}\right) d p_{x} d p_{y}
$$

This is called the impulse approximation. The momentum density $n\left(p_{x}, p_{y}, p_{z}\right)$ is that of the quantum system, not a classical one. In the case of a layered material, such as graphite, $n\left(p_{x}, p_{y}, p_{z}\right)$ is anisotropic and the projection of this density on the momentum-transfer direction will depend on the orientation of $\boldsymbol{q}$. In practice $J(y)$ is presented by a Gaussian with a width that is determined by the mean-kinetic energy due to the momentum component along the recoil direction. For large but finite $q$, one can describe the dynamical structure factor as the sum of $S_{\infty}(\boldsymbol{q}, \omega)$ and correction terms, ${ }^{9}$ 
TABLE I. Parameters, as determined by Fielding et al. (Ref. 5) from neutron scattering, used here to calculate the electron scattering and photoemission line shapes using Eq. (9) are reproduced in the first line. The second line shows the mean kinetic-energy values obtained from the phonon-dispersion model used to determine the line shape by evaluating Eqs. (4) and (6), as was done in Ref. 1.

\begin{tabular}{cccc}
\hline \hline $\begin{array}{c}\left\langle E_{\mathrm{kin}}\right\rangle \perp \\
(\mathrm{eV})\end{array}$ & $\begin{array}{c}\left\langle E_{\mathrm{kin}}\right\rangle \| \\
(\mathrm{eV})\end{array}$ & $\begin{array}{c}\Delta V \perp \\
\mathrm{eV}^{-2}\end{array}$ & $\begin{array}{c}\Delta V \| \\
\mathrm{eV}^{-2}\end{array}$ \\
\hline 0.019 & 0.037 & 36 & 200 \\
0.017 & 0.027 & & \\
\hline \hline
\end{tabular}

$$
S(\boldsymbol{q}, \omega)=\frac{M}{q}\left[1+\sum_{n=3}^{\infty}(-1)^{n} A_{n}(q) \frac{d^{n}}{d y^{n}}\right] J(y),
$$

with the first $A_{n}$ contribution given by $(n=3) \quad A_{3}$ $=M\langle\Delta V\rangle / 36 q, V$ as the total potential energy and $\Delta$ as the Laplacian with respect to the position of the scatterer. The contribution of the correction term increases with decreasing $\boldsymbol{q}$. The correction term shifts the peak to lower energy-loss values. This is consistent with the observations for lowmomentum transfer in Fig. 1 where the observed recoil shift is smaller than that calculated for scattering from a free particle $\left(q^{2} / 2 M\right)$. Subsequent terms in Eq. (9) contain higher derivatives of $J(y)$ and will thus oscillate faster with $\omega$. Hence very good energy resolution is required to observe their contribution.

Neutron scattering estimates of both $J(y)$ and $A_{3}$ exists for graphite, both for $\mathbf{q}$ along and perpendicular to the graphite planes $^{5}$ (see Table I).

We have obtained an analytic expression of Eq. (6) for harmonic crystals, in which anisotropic Debye model is used to reflect a two-dimensional crystal structure of graphite. ${ }^{1,21}$ We will now try to describe all measurements both by direct evaluation of Eqs. (4) and (6), and in terms of the impulse approximation plus a correction term [Eq. (9)].

\section{COMPARISON WITH EXPERIMENT DATA}

The $\left(e, e^{\prime}\right)$ measurements were done at $40 \mathrm{keV}$ and a scattering angle of $44.3^{\circ}(|q|=41.7$ a.u.), and are shown in the left panel of Fig. 2. These thin samples were made by cleavage of HOPG, followed by oxygen/argon plasma etching. (For details see Ref. 2.) The HOPG samples obtained in this way were studied in the past by $(e, 2 e)$ spectroscopy and show an anisotropic electronic structure, as expected for samples with a well-defined orientation of the $c$ axis. ${ }^{22}$ As the zero point of the energy-loss scale is somewhat dependent on sample alignment, etc., a small amount of Au was added to the sample. This provides us with a separate peak, which is (due to the large mass of $\mathrm{Au}$ ) very close to zero energy loss. We assume here that the Au peak is at a recoil energy $q^{2} / 2 M_{\mathrm{Au}}$. The recoil in these $\left(e, e^{\prime}\right)$ experiments is the same as would be experienced by a carbon $1 s$ photoemission using $23.3 \mathrm{keV}$ photons. Hence the calculated shape for this (hypothetical) $(\gamma, e)$ experiment should coincide with the observed line shape of the $\left(e, e^{\prime}\right)$ experiment. This "photo- emission line shape" is obtained by directly evaluating Eqs. (4) and (6), as described in Ref. 1, and using the same model for the phonon density of states. This calculation is compared to the calculated line shape based on Eq. (9) using parameters determined by published neutron-scattering experiments, ${ }^{5}$ which are reproduced in Table I. Both calculations are broadened with the experimental (Gaussian) energy resolution of $\approx 0.35 \mathrm{eV}$. The calculations as well as the measurement were done for $\boldsymbol{q}$ along ("in plane") and perpendicular to the graphite planes. Agreement between the calculations is quite good; both predicting that the peak should be broader when $\boldsymbol{q}$ is along the graphite planes, compared to the peak when it is perpendicular to the planes. There is also an apparent shift in the peak position between both orientations in both theories. The smaller recoil shift is found for $\boldsymbol{q}$ along the graphite planes. In this direction the bonding is strong, hence, substantial deviations of the recoil energy from that of scattering from a free particle are expected. The $\left(e, e^{\prime}\right)$ experiment shows all of the same tendencies. The main deviation is a somewhat larger width for the in-plane measurement.

The photoemission data are shown in the top central panel and the calculated line shape using both approaches in the bottom. For the photon energy used $(h \nu=7940 \mathrm{eV}),|q|$ is smaller (23.8 a.u.) than in the electron-scattering experiment, and hence, corrections to the impulse approximation are more significant. The calculation is now convoluted with a Lorentzian [160 meV full width at half maximum (FWHM)], to represent the core-level lifetime broadening and a Gaussian corresponding the experimental resolution $(120 \mathrm{meV}$ FWHM). Again both calculations are qualitatively in agreement but the line shape obtained from the impulse approximation plus correction is again somewhat broader than the photoemission theory based on evaluating Eq. (6). The experimentally obtained dependence on the target orientation is reproduced by both calculations.

The neutron experiment by Fielding et al. ${ }^{5}$ contained data for one detector (at 53.2 $2^{\circ}$ using an Au filter with a resonance at $4903 \mathrm{meV}$; see, e.g., Ref. 11 for experimental details) that has a very similar momentum transfer (23.4 a.u.) to the photoemission experiment (23.8 a.u.). Hence it is instructive to compare the spectra for this detector as well. This is done in the right panel. Neglecting the small difference in momentum transfer, these spectra should be directly comparable to the $7940 \mathrm{eV}$ photoemission data and indeed the obtained shapes are very similar.

A more direct comparison between both line shapes is given in Fig. 3. The best comparison is obtained if the photoemission recoil energy-loss scale is shifted by $\approx 25 \mathrm{meV}$ relative to the neutron recoil scale. A large part of this shift $(20 \mathrm{meV})$ can be explained if we assume that the Au Fermi level used for the energy callibration ${ }^{1}$ is recoil shifted as well by $q^{2} / 2 M_{\mathrm{Au}}$. The agreement between the two very different experiments is striking. The neutron experiment has somewhat more intense "wings," mainly due to the Lorentzian width of the Au resonance used (258 meV FWHM), which is larger than the lifetime broadening affecting the $\mathrm{C} 1 \mathrm{~s}$ photoemission line (120 meV FWHM). Good agreement is found for both orientations of the recoil momentum.

In contrast to $\left(e, e^{\prime}\right)$ and $(\gamma, e)$ experiments, which measure $S(\boldsymbol{q}, \omega)$ along a line of constant $\boldsymbol{q}$ in the $\left(n, n^{\prime}\right)$ case, $\boldsymbol{q}$ 

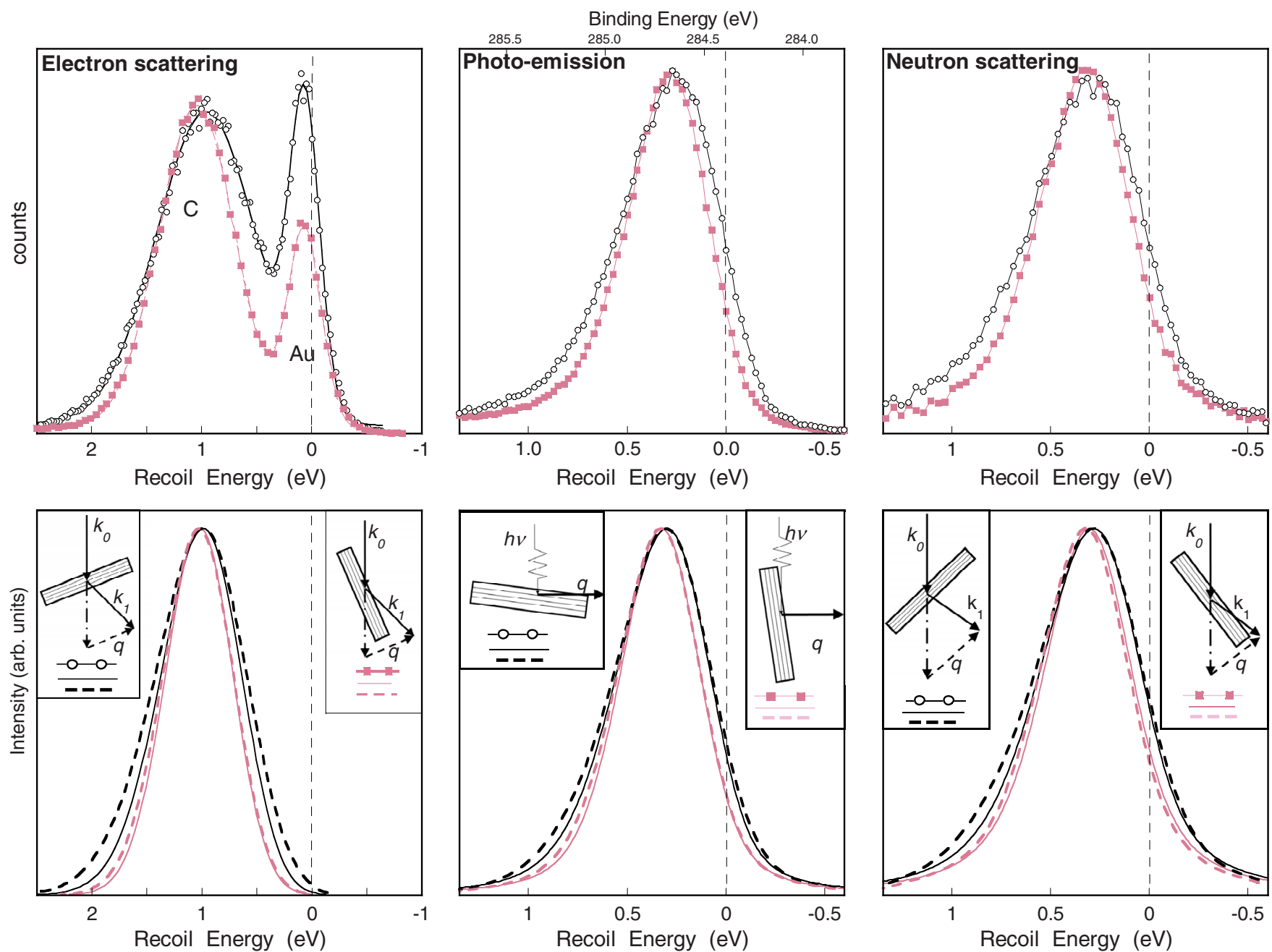

FIG. 2. (Color online) The top-left plot shows the observed spectra for electrons scattering from a thin HOPG sample with momentum transfer $\boldsymbol{q}$ perpendicular (filled squares) to and along (open circles) the graphite planes. The top-central plot shows photoemission results in the same way and neutron-scattering data are shown in the top-right. Here thick HOPG samples were used. In the lower plots we show the calculated line shapes derived either by direct evaluation of Eqs. (4) and (6) (solid lines), and by using the impulse approximation plus corrections [Eq. (9)] with the parameters as determined from neutron scattering by Fielding et al. (Ref. 5) (dashed lines).

depends slightly on the energy of the scattered neutron as the momentum of the detected neutron changes somewhat with its energy. Hence quantitative comparison of both data involves more than just correcting for the different Gaussian and Lorentzian widths.

\section{CONCLUSION AND DISCUSSION}

We have seen that recoil effects affect epithermal-neutron scattering, high-energy electron scattering, and high-energy photoemission in a similar way. Furthermore, all techniques resolve the anisotropy of the atomic motion in graphite and peak shifts due to final-state effects. The high-energy parametrization developed by Sears is an effective way of characterizing the peak shapes over a large range of $\boldsymbol{q}$ values. The small difference between the calculated line shapes using either Eq. (9) or Eqs. (4) and (6) is due to slightly different phonon densities of states (assumed in Ref. 1 and determined experimentally in Ref. 5). This is also evident from the slightly different kinetic energy along and perpendicular to the planes in both cases (see also Table I).

We want to stress once more that both approaches [series expansion of Sears and direct evaluation of Eq. (4) and (6)] are equivalent. The Sears approach highlights that, at highmomentum transfer when the first term dominates, the observed profile is simply a Compton profile of the momentum distribution of the atoms, and stresses the clear link between observed width and mean-kinetic energy of the atoms. At intermediate momentum transfer, one has only to consider the first two terms of the Sears expansion. Thus the Sears method makes it possible to recover the Compton profile even at intermediate momentum transfer. Direct evaluation of Eqs. (4) and (6) should, at any momentum transfer, describe the experiment correctly and the results should always be the same as the Sears expansion provided one includes sufficient terms.

Each technique has its own characteristics for the study of recoil effects. For example, neutron scattering measures true bulk properties while photoemission will, in a compound 


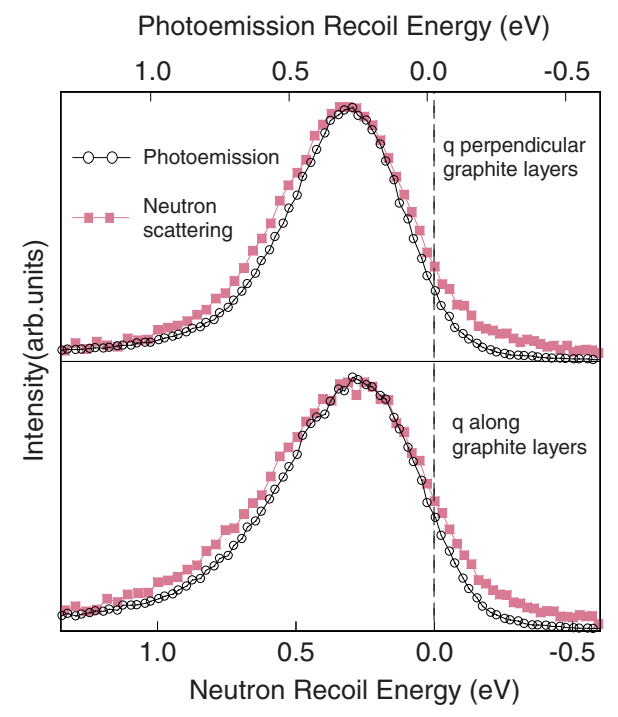

FIG. 3. (Color online) A comparison between photoemission (open circles) and neutron scattering (filled squares) spectra obtained at similar momentum transfers. The top panel is for $\mathbf{q}$ along the graphite planes while the bottom panel is for $\mathbf{q}$ perpendicular to the graphite planes. The photoemission recoil scale is shifted by 25 $\mathrm{meV}$ for reasons explained in the main text.

sample, completely separate the profiles of different elements. Photoemission can provide element specific profiles. Electron scattering requires comparatively small equipment and can be done "in house." Thus the preferred technique to study $S(\boldsymbol{q}, \omega)$ depends on the material and $\boldsymbol{q}$ values of interest.

A paper by Fujikawa et al. describes photoemission line shapes in terms of recoil and the Franck-Condon effect. ${ }^{23}$ The agreement with the neutron measurements seems to indicate that the influence of the Franck-Condon effect on the line shape is relatively minor. In a very recent paper, Fujikawa $e t$ al. explored the recoil effect beyond the singlesite approximation. ${ }^{24}$ There is, in our experimental data, no evidence that the incoherent (or single-site) approximation does not suffice to describe the data.

In conclusion it is clear that recoil effects have a similar influence on all three spectroscopies described here. In particular this means that interpretation of the recoil effect in photoemission and electron scattering can benefit greatly from the study of the well-developed theory for the case of neutron scattering. Finally it is worth mentioning that, for weakly bound systems (liquid Ne), similar recoil effects have been observed using $\mathrm{x}$-ray scattering. ${ }^{25}$

\section{ACKNOWLEDGMENTS}

We thank members of Coherent X-ray Optics Laboratory and Excitation Order Research Team in the RIKEN SPring-8 Center for valuable discussions and encouragements. This work was made possible by a grant of the Australian Research Council and was partially supported by the Ministry of Education, Science, Sports and Culture of Japan through a Grant-in-Aid for Scientific Research (Grants No. 15206006, No. 18540323, and No. 17340097).
${ }^{1}$ Y. Takata et al., Phys. Rev. B 75, 233404 (2007).

${ }^{2}$ M. Vos and M. R. Went, Phys. Rev. B 74, 205407 (2006).

${ }^{3}$ H. Rauh and N. Watanabe, Phys. Lett. 100A, 244 (1984).

${ }^{4}$ M. P. Paoli and R. S. Holt, J. Phys. C 21, 3633 (1988).

${ }^{5}$ A. L. Fielding, D. N. Timms, and J. Mayers, Europhys. Lett. 44, 255 (1998).

${ }^{6}$ W. E. Lamb, Phys. Rev. 55, 190 (1939).

${ }^{7}$ L. van Hove, Phys. Rev. 95, 249 (1954).

${ }^{8} \mathrm{G}$. Squires, Introduction to the Theory of Thermal Neutron Scattering (Cambridge University Press, Cambridge, 1978).

${ }^{9}$ V. F. Sears, Phys. Rev. B 30, 44 (1984).

${ }^{10}$ G. I. Watson, J. Phys.: Condens. Matter 8, 5955 (1996).

${ }^{11}$ C. Andreani, D. Colognesi, J. Mayers, G. F. Reiter, and R. Senesi, Adv. Phys. 54, 377 (2005).

${ }^{12}$ P. C. Hohenberg and P. M. Platzman, Phys. Rev. 152, 198 (1966).

${ }^{13}$ A. L. Fielding, D. N. Timms, and J. Mayers, Physica B (Amsterdam) 276-278, 69 (2000).

${ }^{14}$ H. Boersch, R. Wolter, and H. Schoenebeck, Z. Phys. 199, 124 (1967).
${ }^{15}$ D. Varga, K. Tökési, Z. Berényi, J. Tóth, L. Körvér, G. Gergely, and A. Sulyok, Surf. Interface Anal. 31, 1019 (2001).

${ }^{16}$ M. Went and M. Vos, Appl. Phys. Lett. 90, 072104 (2007).

${ }^{17}$ M. Vos and M. Went, Surf. Sci. 601, 4862 (2007).

${ }^{18}$ K. Siegbahn et al., Nova Acta Regiae Soc. Sci. Ups. 20, 35 (1967).

${ }^{19}$ W. S. M. Werner, C. Tomastik, T. Cabela, G. Richter, and H. Störi, J. Electron Spectrosc. Relat. Phenom. 113, 127 (2001).

${ }^{20}$ M. Vos and M. R. Went, Surf. Sci. 601, 1536 (2007).

${ }^{21}$ J. Gunn and M. Warner, Z. Phys. B: Condens. Matter 56, 13 (1984).

${ }^{22}$ T. Sattler, T. Tschentscher, J. R. Schneider, M. Vos, A. S. Kheifets, D. R. Lun, E. Weigold, G. Dollinger, H. Bross, and F. Bell, Phys. Rev. B 63, 155204 (2001).

${ }^{23}$ T. Fujikawa, R. Suzuki, and J. Kövér, J. Electron Spectrosc. Relat. Phenom. 151, 170 (2006).

${ }^{24}$ T. Fujikawa, H. Arai, R. Suzuki, H. Shinotsuka, L. Kövér, and N Ueno, J. Electron Spectrosc. Relat. Phenom. 162, 146 (2008).

${ }^{25}$ G. Monaco, A. Cunsolo, G. Pratesi, F. Sette, and R. Verbeni, Phys. Rev. Lett. 88, 227401 (2002). 